\title{
GENES ENCODING PROTEINS REgUlating FATTY ACID METABOLISM AND CELLULAR RESPONSE TO LIPIDS ARE DIFFERENTIALLY EXPRESSED IN PORCINE LUMINAL EPITHELIUM DURING LONG-TERM CULTURE
}

Magdalena Kulus ${ }^{1,4}$, Blanka Borowiec ${ }^{1,2}$, Małgorzata Popis ${ }^{1}$, Piotr Celichowski ${ }^{2}$, Michal Jeseta ${ }^{3}$, Dorota Bukowska ${ }^{4}$, Hanna Piotrowska-Kempisty ${ }^{5}$, Małgorzata Bruska ${ }^{1}$, Maciej Zabel ${ }^{6,7}$, Michał Nowicki $^{2}$, Bartosz Kempisty ${ }^{1,2,3}$, Paweł Antosik ${ }^{4}$

\begin{abstract}
Among many factors, the epithelium lining the oviductal lumenis very important for the development of the oocyte and its subsequent fertilization. The oviductal epithelium is characterized by the presence of ciliary cells, supporting the movement of cumulus-oocyte complexes towards the uterus. By interacting with the semen, the epithelium of the fallopian tube makes the sperm acquire the ability to fertilize. So far, the exact molecular mechanisms of these changes have not been known. Hence, understanding the metabolism of oviduct epithelial cells and the level of expression of individual groups of genes seems to be a way to deepen the knowledge about the broadly understood reproduction.

In our research, we decided to culture oviductal epithelial cells (OECs) in vitro for a long period of time. After 24h, 7, 15 and 30 days, the OECs were harvested, with their RNA isolated. Transcriptomic changes were analyzed using microarrays. The "cellular response to lipid" group was represented by the following genes: MUC1, CYP24A1, KLF4, IL24, SNAI2, CXCL10, PPARD, TNC, ABCA10, while the genes belonging to the "cellular lipid metabolic processes" were: LIPG, ARSK, ACADL, FADS3, P2RX7, ACSS2, PPARD, KITLG, SPTLC3, ERBB3, KLF4, CRABP2. Additionally, PPARD and ACADL were members of the "fatty acid beta-oxidation" ontology group. Our study describes genes that are not directly related to fertility processes. However, significant changes in their expression in in vitro cultured OECs may indicate their usefulness as markers of OECs' physiological processes.
\end{abstract}

Running title: Fatty acids changes in porcine oviductal epithelial cells in in vitro cultivation

Keywords: porcine oviduct epithelial cells, fatty acids, in vitro maturation (IVM), microarrays

\footnotetext{
${ }^{1}$ Department of Anatomy, Poznan University of Medical Sciences, Poznan, Poland

${ }^{2}$ Department of Histology and Embryology, Poznan University of Medical Sciences, Poznan, Poland

${ }^{3}$ Department of Obstetrics and Gynecology, University Hospital and Masaryk

University, Brno, Czech Republic

${ }^{4}$ Veterinary Centre, Nicolaus Copernicus University in Torun, Torun, Poland

${ }^{5}$ Department of Toxicology, Poznan University of Medical Sciences, Poznan, Poland

${ }^{6}$ Department of Histology and Embryology, Wroclaw Medical University, Wroclaw, Poland

${ }^{7}$ Division of Anatomy and Histology, University of Zielona Gora, Zielona Góra, Poland

* Correspondence: bkempisty@ump.edu.pl

Full list of author information is available at the end of article
} 


\section{Introduction}

Fertility is a complicated phenomenon success of which is influenced by many factors. It is known that oocytes mature gradually, with only oocytes maintained under excellent conditions are able to progress past the MII stage [1]. Many studies focus on factors determining the reproductive capacity of oocytes, expression of their genes and in vitro maturation. It is obvious that oocytes play a key role here, but if it were not for the mechanisms surrounding the ovum, it would be impossible to obtain the right conditions for fertilization [2]. Among many factors, the epithelium lining the lumen of the fallopian tubes is very important for the development of the oocyte and its subsequent fertilization [3]. The oviductalepithelium is characterized by the presence of ciliary cells, supporting the movement of cumulus-oocyte complexes towards the uterus. By interacting with the semen, the epithelium of the fallopian tube allows the sperm to acquire the ability to fertilize [4]. The transport of spermatozoa through the female reproductive tract seemsto be an especially important stage [5]. So far, the exact molecular mechanisms of these changes have not been discovered. Hence, understanding the metabolism of oviduct epithelial cells and the level of expression of individual groups of genes seems to be a way to deepen the knowledge about the broadly understood reproduction.

The aim of our research was to maintain the culture of epithelial cells of the porcine fallopian tube in primary culture. We carried out this culture over a long term, lasting up to 30 days. We have made observations of biochemical and morphological changes occurring during the cell culture. We analyzed the expression profile of genes responsible for regulating the metabolism of fatty acids and cellular response to lipids. As there are reports of the negative effects of non-esterified fatty acids (NEFA) on the reproductive system $[5,6]$, we decided to study their metabolism in oviductal epithelial cells (OECs).

\section{Material and methods \\ Animals}

In this study, crossbred gilts $(n=45)$ at the age of about nine months and which displayed at least two regular estrous cycles were collected from a commercial herd. All the animals were checked daily for estrus behavior and were slaughtered after reaching the anestrus phase of the estrus cycle. The uteri were then transported to the laboratory within 30 $\min$ at $38^{\circ} \mathrm{C}$.

\section{Oviductal epithelial cells (OECs) selection and culture}

Oviducts were washed twice in Dulbecco's phosphate buffered saline (PBS) (137 mM NaCl, $27 \mathrm{mM}$ $\mathrm{KCl}, 10 \mathrm{mM} \mathrm{Na} 2 \mathrm{HPO} 4,2 \mathrm{mM} \mathrm{KH} 2 \mathrm{PO} 4, \mathrm{pH}$ 7.4). Epithelial cells were removed using sterile surgical blades. Then, the epithelium was incubated with collagenase I (Sigma Aldrich, Madison, USA), 1mg/ $\mathrm{mL}$ in Dubecco's modified Eagle's medium (DMEM; Sigma Aldrich, Madison, USA) for $1 \mathrm{~h}$ at $37 \mathrm{oC}$. The cell suspension obtained from this digestion was filtered through $40 \mu \mathrm{m}$ pore size strainer to remove blood and single cells. The residue was collected by rinsing the strainer with DMEM. The cells were then centrifuged ( $200 \mathrm{x} \mathrm{g}, 10 \mathrm{~min}$.). Next, they were washed in PBS and centrifuged again. Later, they were incubated with $0.5 \%$ Trypsin/EDTA (Sigma Aldrich, Madison, USA) at 37oC for $10 \mathrm{~min}$. The reaction was stopped with fetal calf serum (FCS; Sigma Aldrich, Madison, USA). After incubation, the cells were filtered and centrifuged for the last time. The final cell pellet was suspended in DMEM, supplemented with $10 \% \mathrm{FCS}, 100 \mathrm{U} / \mathrm{mL}$ penicillin, $100 \mu \mathrm{g} / \mathrm{mL}$ streptomycin and $1 \mu \mathrm{g} / \mathrm{mL}$ amphotericin B. The cells were cultured at $37^{\circ} \mathrm{C}$ in a humidified atmosphere of $5 \% \mathrm{CO} 2$. Once the OEC cultures attained $70-80 \%$ confluency, they were passaged by washing with PBS, digested with $0.025 \%$ Trypsin/ EDTA, neutralized by a $0.0125 \%$ trypsin inhibitor (Cascade Biologics, Portland, USA), centrifuged, and resuspended at a seeding density of $2 * 10^{4}$ cells/ $\mathrm{cm} 2$. The culture medium was changed every three days. The culture was maintained for 30 days.

\section{RNA extraction from oviductal epithelial cells (OECs)}

Oviductal epithelial cell were pooled and harvested $24 \mathrm{~h}, 7$ days, 15 days and 30 days after the beginning of culture. Total RNA was extracted from the samples using TRI Reagent (Sigma, St Louis, MO, USA) and RNeasy MinElute cleanup Kit (Qiagen, Hilden, Germany). The total mRNA amount was determined from the optical density at $260 \mathrm{~nm}$, and the RNA purity was estimated using the $260 / 280$ $\mathrm{nm}$ absorption ratio (higher than 1.8) (NanoDrop spectrophotometer, Thermo Scientific, ALAB, Poland). The RNA integrity and quality were checked on a Bioanalyzer 2100 (Agilent Technologies, Inc., Santa Clara, CA, USA). The resulting RNA integrity numbers (RINs) were between 8.5 and 10 with an average of 9.2 (Agilent Technologies, Inc., Santa Clara, CA, USA). The RNA in each sample was diluted to a concentration of $100 \mathrm{ng} / \mu \mathrm{l}$ with an OD260/ OD280 ratio of 1.8/2.0. From each RNA sample, $100 \mathrm{ng}$ of RNA was taken for microarray expression assays.

\section{Microarray expression analysis and statistics}

Total RNA (100 ng) from each pooled sample was subjected to two rounds of sense cDNA amplification (Ambion ${ }^{\circledR}$ WT Expression Kit). The obtained cDNA was used for biotin labeling and fragmentation using Affymetrix GeneChip® WT Terminal Labeling and Hybridization (Affymetrix, Santa Clara, CA, USA). Biotin-labeled fragments of cDNA (5.5 $\mu \mathrm{g})$ 
were hybridized to the Affymetrix $\AA$ Porcine Gene 1.1 ST Array Strip $\left(48^{\circ} \mathrm{C} / 20 \mathrm{~h}\right)$. Microarrays were then washed and stained, according to the technical protocol, using the Affymetrix GeneAtlas Fluidics Station. The array strips were scanned employing the Imaging Station of the GeneAtlas System. Preliminary analysis of the scanned chips was performed using Affymetrix GeneAtlas ${ }^{\mathrm{TM}}$ Operating Software. The quality of gene expression data was confirmed according to the quality control criteria provided by the software. The obtained CEL files were imported into downstream data analysis software.

All of the presented analyses and graphs were compiled using Bioconductor and R programming languages. Each CEL file was merged with a description file. To correct background, normalize, and summarize results, we used the Robust Multiarray Averaging (RMA) algorithm. To determine the statistical significance of the analyzed genes, moderated t-statistics from the empirical Bayes method were performed. The obtained p-value was corrected for multiple comparisons using Benjamini and Hochberg's false discovery rate. Selection of significantly altered genes was based on a p-value beneath 0.05 and expression higher than two-fold.

Differentially expressed genes were subjected selection by examination of genes involved in oxygen metabolism. The differentially expressed gene list (separated for up- and down-regulated genes) was uploaded to the DAVID software (Database for Annotation, Visualization and Integrated Discovery) [7], where genes belonging to the terms of all four Gene Ontologies (GOs) of interest were extracted. Expression data of these genes was also subjected to a hierarchical clusterization procedure, with their expression values presented as a heat map.

Subsequently, we analyzed the relation between the genes belonging to the chosen GO terms using theGOplot package [8]. The GoPlot package had calculated the z-score: the number of up-regulated genes minus the number of down-regulated genes divided by the square root of the count. This information allowed to estimate the change course of each gene-ontology term.

Interactions between differentially expressed genes/proteins belonging to the studied gene ontology groups were investigated by the STRING10 software (Search Tool for the Retrieval of Interacting Genes) [9]. The list of gene names was used as a query for interaction prediction. The search criteria were based on co-occurrences of genes/proteins in scientific texts (text mining), co-expression, and experimentally observed interactions. The results of such analyses generated a gene/protein interaction network where the intensity of the edges reflected the strength of the interaction score.

Finally, the functional interactions between genes that belongs to the chosen GO BP terms were investigated by the REACTOME FIViz application to the
Cytoscape 3.6.0 software. The ReactomeFIViz app is designed to find pathways and network patterns related to cancer and other types of diseases. This app accesses the pathways stored in the Reactome database, allowing to perform pathway enrichment analysis for a set of genes, visualize hit pathways using manually laid-out pathway diagrams directly in Cytoscape, and investigate functional relationships among genes in hit pathways. The app can also access the Reactome Functional Interaction (FI) network, a highly reliable, manually curated pathway-based protein functional interaction network covering over $60 \%$ of human proteins.

\section{Ethical approval}

The research related to animal use has been complied with all the relevant national regulations and instructional policies for the care and use of animals. Bioethical Committee approval no. 83/2012/DNT.

\section{Results}

Whole transcriptome profiling with Affymetrix microarrays allows us to analyze the gene expression changes between 7,15 and 30 days of porcine oviductal epithelial cell culture. Using Affymetrix $®$ Porcine Gene 1.1 ST Array Strip, we have examined the expression of 12257 transcripts. Genes with fold change higher than abs (2) and with corrected $p$-value lower than 0.05 were considered as differentially expressed. This set of genes consists of 2533 different transcripts.

DAVID (Database for Annotation, Visualization and Integrated Discovery) software was used for extraction of gene ontology biological process terms (GO BP) that contain differently expressed transcripts. Up and down regulated gene sets were subjected to the DAVID search separately and only gene sets with adj. p-value lower than 0.05 were selected. The DAVID software analysis showed that the differently expressed genes belonged to 657 Gene ontology terms. In this paper, we focused on 166 genes that belong to "cellular lipid metabolic process", "cellular response to lipid" and "fatty acid beta-oxidation"GO BP terms. These sets of genes were subjected to hierarchical clusterization procedure and presented as heatmaps (Fig. 1). The gene symbols, fold changes in expression, Entrez gene IDs and corrected p-values of these genes were shown in table 1.

The enrichment of each GO BP term was calculated as a z-score and shown on the circle diagram (Fig. 2).

The chosen GO BP terms contain 166 differently expressed genes. Therefore, we calculated the mean fold change ratio value of each gene between 7,15 and 30 days of culture. Based on that criteria, we chose the 10 most downregulated and 10 most upregulated genes for further analysis.

In Gene Ontology database, genes that form one particular GO can also belong to other GO term categories. For this reason, we explored the gene in- 

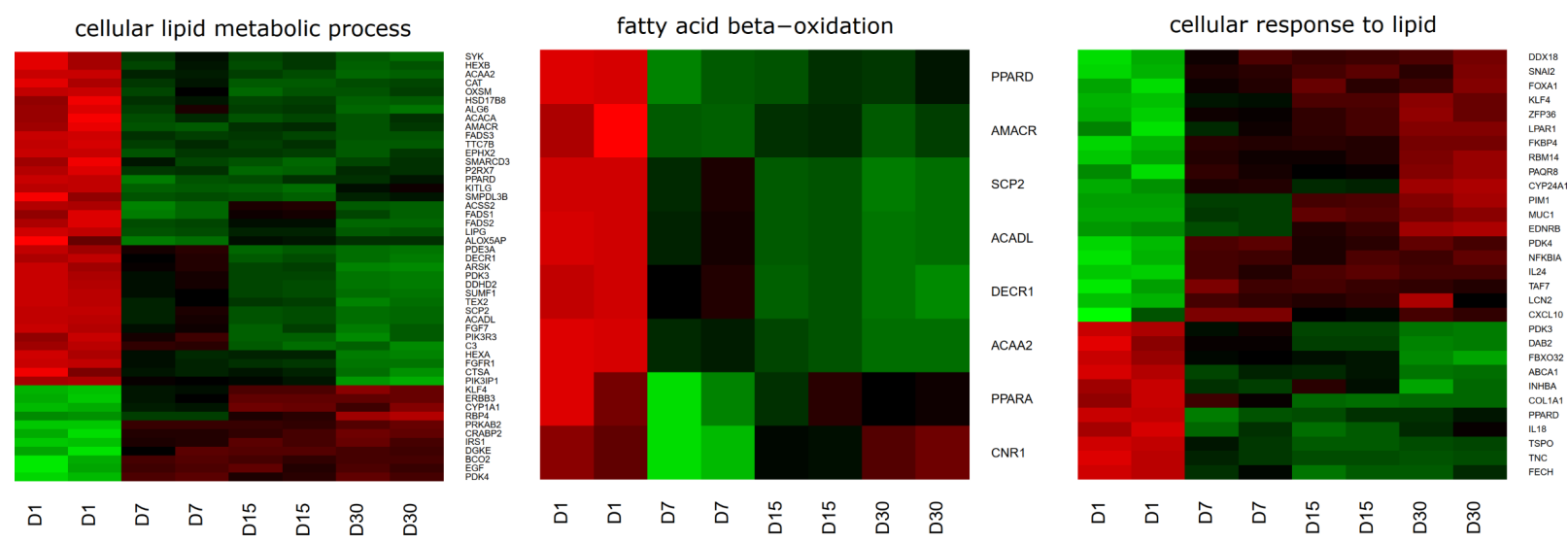

FIGURE 1 Heat map representation of differentially expressed genes belonging to the to "cellular lipid metabolic process", "cellular response to lipid" and "fatty acid beta-oxidation" GO BP terms. Arbitrary signal intensity acquired from microarray analysis is represented by colours (green, higher; red, lower expression). Log2 signal intensity values for any single gene were resized to Row Z-Score scale (from -2 , the lowest expression to +2 , the highest expression for single gene)

TABLE 1 Gene symbols, fold changes in expression, corrected p values and Entrez gene IDs of studied genes

\begin{tabular}{|c|c|c|c|c|c|c|}
\hline $\begin{array}{c}\text { GENE } \\
\text { SYMBOL }\end{array}$ & $\begin{array}{c}\text { FOLD } \\
\text { CHANGE } \\
\text { D7/D1 } \\
\end{array}$ & $\begin{array}{c}\text { FOLD } \\
\text { CHANGE } \\
\text { D15/D1 } \\
\end{array}$ & $\begin{array}{c}\text { FOLD } \\
\text { CHANGE } \\
\text { D30/D1 } \\
\end{array}$ & $\begin{array}{c}\text { ADJUSTED P } \\
\text { VALUE D7/D1 }\end{array}$ & $\begin{array}{c}\text { ADJUSTED P } \\
\text { VALUE D15/D1 }\end{array}$ & $\begin{array}{c}\text { ADJUSTED P } \\
\text { VALUE D30/D1 }\end{array}$ \\
\hline IL24 & 0,083371 & 0,062192 & 0,071399 & $9,50 \mathrm{E}-06$ & $3,21 \mathrm{E}-06$ & $1,76 \mathrm{E}-06$ \\
\hline CRABP2 & 0,108046 & 0,084638 & 0,056801 & $3,11 \mathrm{E}-05$ & $1,05 \mathrm{E}-05$ & $2,84 \mathrm{E}-06$ \\
\hline СYP24A1 & 0,149169 & 0,294276 & 0,039584 & 2,69E-05 & 0,000136 & $7,91 \mathrm{E}-07$ \\
\hline SNAI2 & 0,183766 & 0,134521 & 0,130079 & 0,000411 & 0,000129 & $7,85 \mathrm{E}-05$ \\
\hline SPTLC3 & 0,191786 & 0,502461 & 0,343172 & $3,75 \mathrm{E}-05$ & 0,001666 & 0,000124 \\
\hline$K L F 4$ & 0,205489 & 0,089904 & 0,057459 & $4,71 \mathrm{E}-05$ & $3,93 \mathrm{E}-06$ & $1,01 \mathrm{E}-06$ \\
\hline MUC1 & 0,240726 & 0,03474 & 0,02121 & $4,67 E-05$ & $9,76 \mathrm{E}-07$ & $2,44 \mathrm{E}-07$ \\
\hline CXCL10 & 0,241713 & 0,447019 & 0,331634 & 0,002165 & 0,025903 & 0,004916 \\
\hline ERBB3 & 0,270208 & 0,12388 & 0,117998 & 4,67E-05 & $3,53 \mathrm{E}-06$ & $1,48 \mathrm{E}-06$ \\
\hline$F K B P 4$ & 0,291215 & 0,291114 & 0,192272 & $4,73 \mathrm{E}-05$ & $2,82 \mathrm{E}-05$ & $4,24 \mathrm{E}-06$ \\
\hline ARSK & 5,76671 & 14,50752 & 31,32185 & 0,000123 & $1,00 \mathrm{E}-05$ & $1,73 \mathrm{E}-06$ \\
\hline KITLG & 5,975557 & 6,478399 & 3,302164 & $1,01 \mathrm{E}-05$ & $3,68 \mathrm{E}-06$ & $1,88 \mathrm{E}-05$ \\
\hline P2RX7 & 6,165142 & 8,522042 & 5,795398 & $2,69 \mathrm{E}-05$ & $6,72 \mathrm{E}-06$ & $1,03 \mathrm{E}-05$ \\
\hline ABCA1 & 6,313396 & 5,556747 & 10,33558 & $3,95 \mathrm{E}-05$ & 3,12E-05 & $4,36 \mathrm{E}-06$ \\
\hline ACSS2 & 6,349718 & 2,503393 & 5,62093 & $7,45 \mathrm{E}-06$ & $9,49 \mathrm{E}-05$ & $2,39 \mathrm{E}-06$ \\
\hline ACADL & 6,552573 & 13,07545 & 16,70385 & $6,68 \mathrm{E}-05$ & 9,39E-06 & $3,46 \mathrm{E}-06$ \\
\hline TNC & 6,57267 & 8,352323 & 8,773467 & $1,83 \mathrm{E}-05$ & $4,91 \mathrm{E}-06$ & $2,42 \mathrm{E}-06$ \\
\hline PPARD & 9,200973 & 6,826522 & 5,602991 & $2,25 \mathrm{E}-05$ & $2,24 \mathrm{E}-05$ & $2,49 \mathrm{E}-05$ \\
\hline FADS3 & 9,516455 & 10,25019 & 12,30064 & $4,27 \mathrm{E}-06$ & $1,47 \mathrm{E}-06$ & $4,44 \mathrm{E}-07$ \\
\hline$L I P G$ & 36,5469 & 19,67815 & 39,01801 & $2,50 \mathrm{E}-06$ & 1,39E-06 & $2,57 \mathrm{E}-07$ \\
\hline
\end{tabular}

tersections between the selected GO BP terms. The relation between those GO BP terms was presented as circle plot (Fig. 3) as well as heatmap (Fig. 4).

STRING interaction network was generated among the differentially expressed genes belonging to each of the selected GO BP terms. Using such prediction method provided us with a molecular interaction network formed between protein products of the studied genes (Fig. 5).
Finally, we investigated the functional interactions between the chosen genes with REACTOME FIViz app to the Cytoscape 3.6.0 software. The results were shown in (Fig. 6).

\section{Discussion}

Increasing food demand observed nowadays is connected with the necessity of more efficient acquisition of raw animal materials. Attempts are be- 
D7/D1

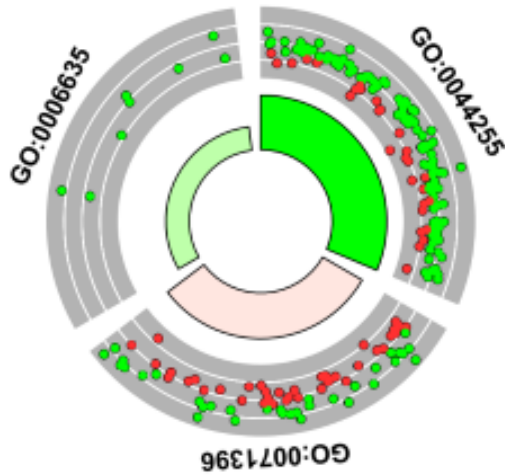

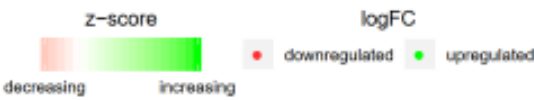

D15/D1

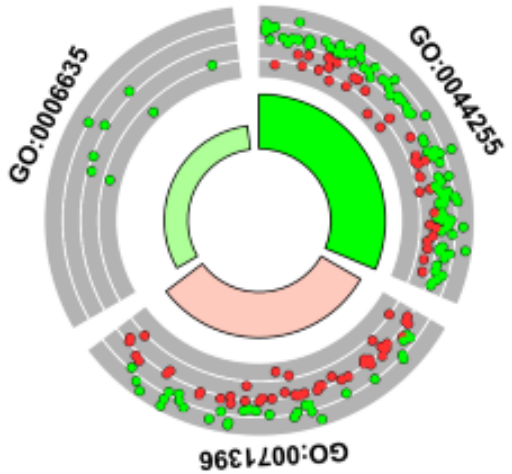

96\&LL00:09
D30/D1

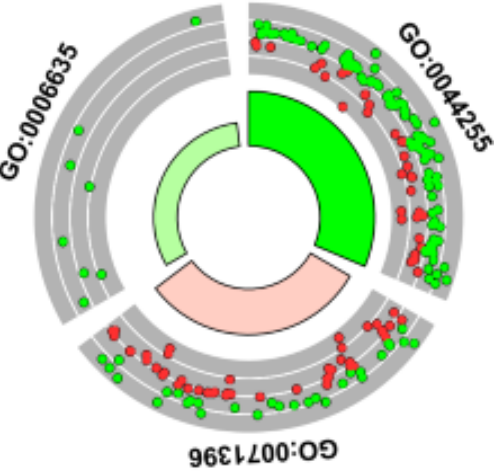

Description

GO:0044255 cellular lipid metabolic process GO:0071396 cellular response to lipid

GO:0006635 fatty acid beta-oxidation

FIGURE 2 The circle plot showing the differently expressed genes and z-score of the "cellular lipid metabolic process", "cellular response to lipid" and "fatty acid beta-oxidation" GO BP terms. The outer circle shows a scatter plot for each term of the fold change of the assigned genes. Green circles display up- regulation and red ones down- regulation. The inner circle shows the z-score of each GO BP term. The width of the each bar corresponds to the number of genes within GO BP term and the color corresponds to the z-score

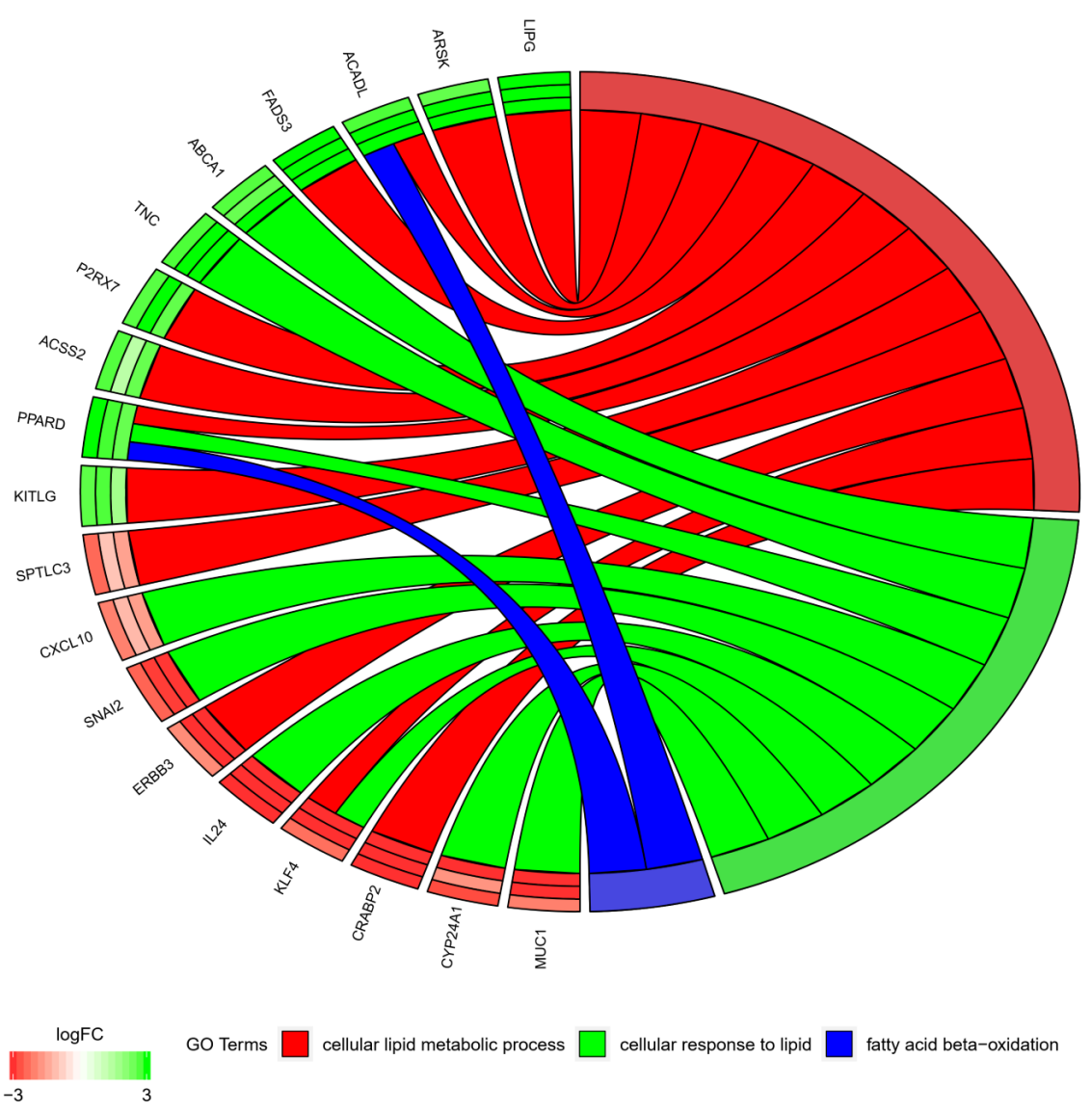

FIGURE 3 The representation of the mutual relationship of differently expressed genes that belongs to the "cellular lipid metabolic process", "cellular response to lipid" and "fatty acid beta-oxidation" GO BP terms. The ribbons indicate which gene belongs to which categories. The middle circle represents logarithm from fold change (LogFC) between D7/D1, D15/D1 and D30/D1 respectively. The color of each block corresponds to the LogFC of each gene (green up-regulated, red - down-regulated). The genes were sorted by logFC from most to least changed gene 


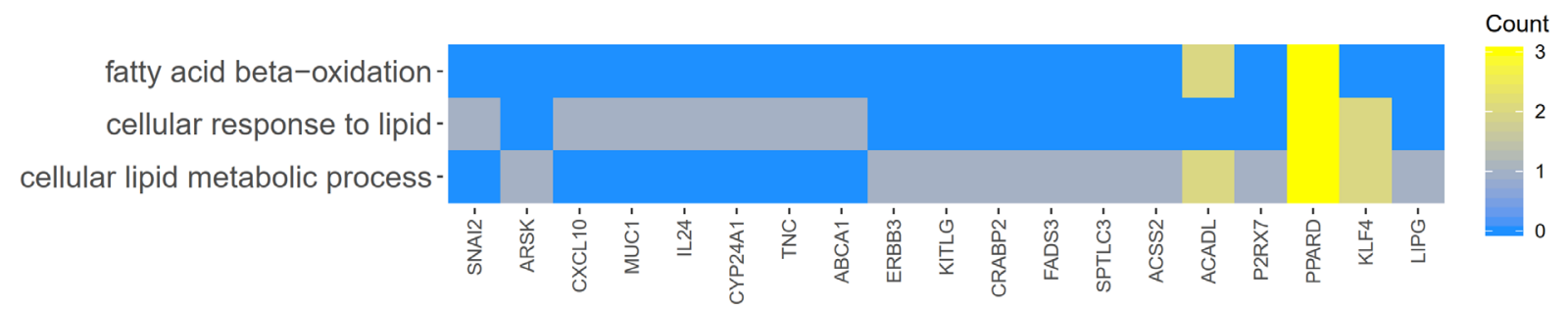

FIGURE 4 Heatmap showing the gene occurrence between differently expressed genes that belongs to the "cellular lipid metabolic process", "cellular response to lipid" and "fatty acid beta-oxidation" GO BP terms. The yellow color is associated with gene occurrence in the GO Term. The intensity of the color is corresponding to amount of GO BP terms that each gene belongs to

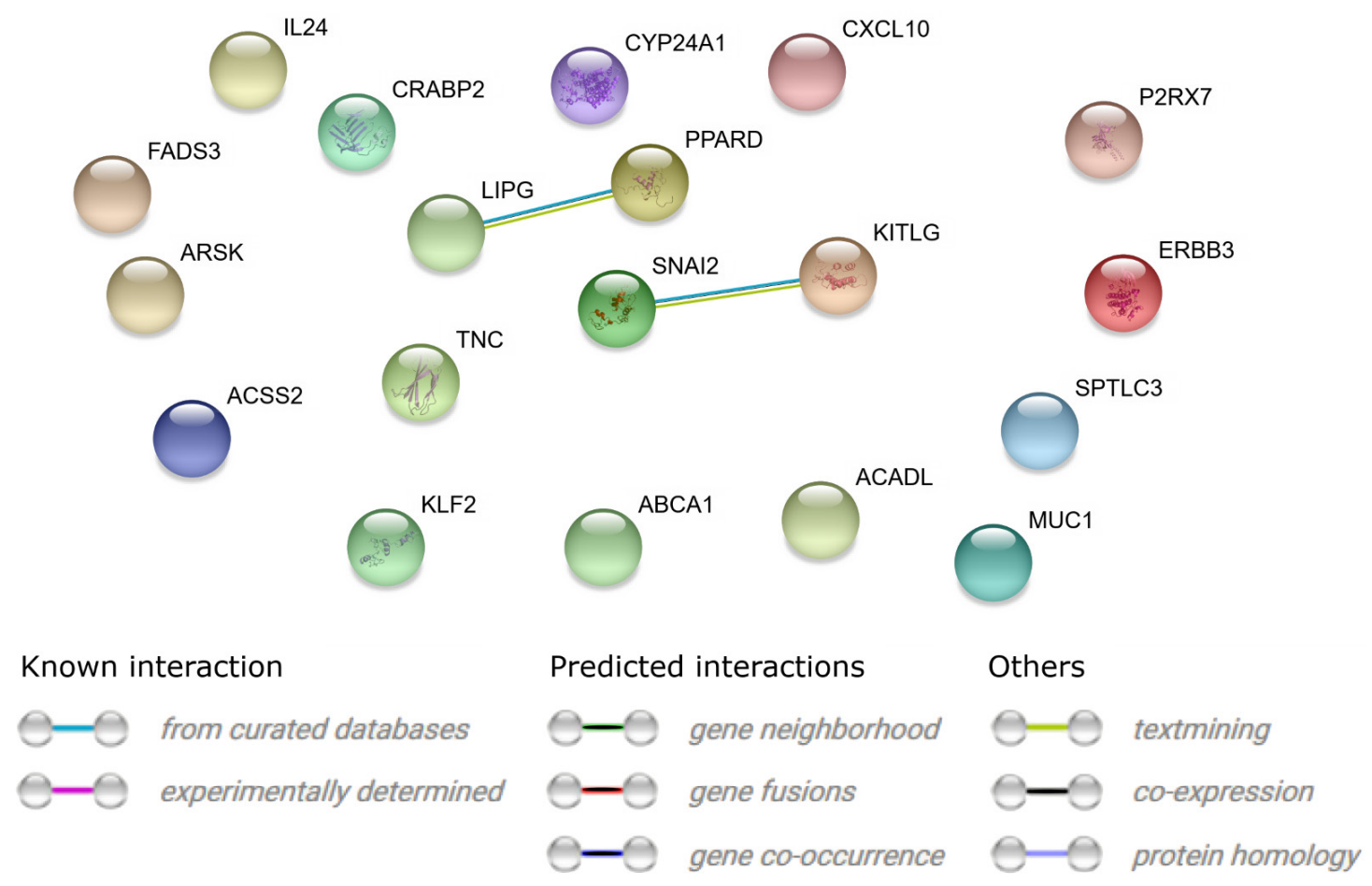

FIGURE 5 STRING-generated interaction occurrence between differently expressed genes that belongs to the "cellular lipid metabolic process", "cellular response to lipid" and "fatty acid beta-oxidation" GO BP terms. The intensity of the edges reflects the strength of interaction score

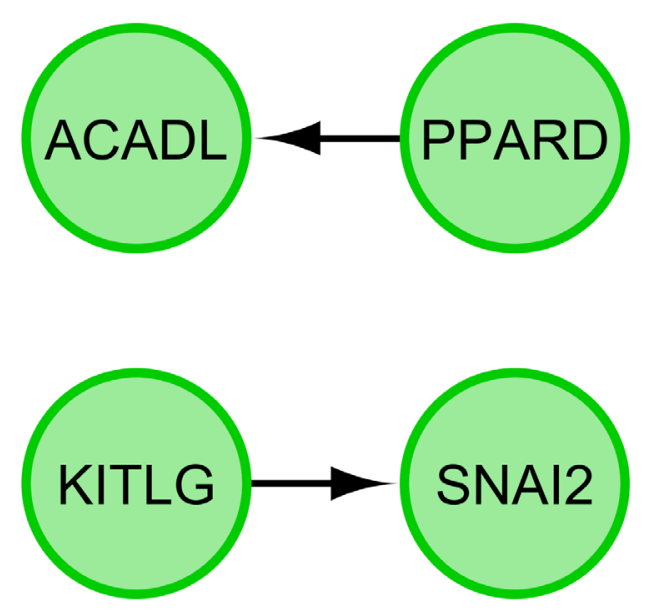

FIGURE 6 Functional interaction (FI) between differently expressed genes that belongs to the "cellular lipid metabolic process", "cellular response to lipid" and "fatty acid beta-oxidation" GO BP terms. In following figure "->" stands for activating/catalyzing, "-|" for inhibition, "-" for FIs extracted from complexes or inputs, and "---" for predicted FIs 
ing made to increase the reproductive capacity of various animal species. Most often, this is achieved through introduction of more caloric diets. The metabolic pathways associated with fat management are also changing. Increasingly, the cause of metabolic disorders is attributed to elevated NEFA levels in the blood. It has also been shown that high values of these fatty acids may have an effect on the deterioration of reproductive rates in animals and humans [10-12].Studies using in vitro cultured bovine OECs have shown that increased NEFA levels may adversely affect the physiology of these cells, but not their morphology. The consequence of these physiological changes may be a decrease in the reproductive capacity of dairy cattle [13]. Recent studies have described the role of fatty acids in the function of sperm in the oviducts of hens [14]. The important role of fatty acids in sperm viability has been proven in this study. On the other hand, other studies carried out on in vitro cultured porcine oocytes showed a significant improvement in both nuclear and cytoplasmic maturation of oocytes after treatment with $100 \mu$ ALA (alpha-linolenic acid) [15].

In our research we focused on genes related to the widely understood metabolism of fatty acids. The results of this study showed genes belonging to three ontological groups, each of which was associated with lipids. Not all these genes are closely related to the reproductive system in pigs, including the fallopian tube. Nevertheless, some of these genes are linked to animal reproductive aspects.

In our in vitro OEC culture studies, we demonstrated significant changes in expression of genes belonging to three main ontological groups: "cellular lipid metabolic processes", "cellular response to lipid" and "fatty acid beta-oxidation". The last-mentioned group included two genes: PPARD and $A C A D L$, which showed up-regulation after in vitro culture (IVC). The PPARD gene (peroxisome proliferator activated receptor delta) participates in fat metabolism, but also has a role in differentiation towards adipocytes [16].

In "Cellular lipid metabolic processes" and "fatty acid beta-oxidation" the general trend of expression was increasing, as the genes showed up-regulation after the first day of culture. The "cellular response to lipid" group, on the other hand, showed more differentiated expression pattern, with a slight down-regulation predominance after the first day of the experiment.

The "cellular response to lipid" group was represented by the following genes: MUC1, CYP24A1, KLF4, IL24, SNAI2, CXCL10, which all showed down-regulation. The remaining 3 genes from this group (PPARD, TNC, ABCA10) showed up-regulation. The above mentioned PPARD gene is a member of all three described groups of genes. The remaining 12 genes form the "cellular lipid metabolic processes" GO, and showed increased (LIPG, ARSK,
ACADL, FADS3, P2RX7, ACSS2, PPARD, KITLG), as well as decreased expression (SPTLC3, ERBB3, KLF4, CRABP2).

The CRABP2 gene (cellular retinoic acid binding protein 2), belonging to the family of lipoproteins, plays a very important role in the metabolism of retinoic acid (RA) and its intracellular transport. Studies conducted on mouse embryos have shown that both $C R A B P 1$ and $C R A B P 2$ transcripts show specific distribution patterns and play an important role in the embryo development processes [17].

Expression of the SPTLC3 gene (serine palmitoyl transferase long chain base subunit 3) leads to the formation of the serine palmitoyl transferase subunit, which takes part in the biosynthesis of sphingolipids [18].

The MUC1 gene expression is responsible for the formation of mucin proteins, which are characteristic for epithelia. Mucins are responsible for creating a barrier on the surface of the epithelium and are also involved in intracellular signal transmission [19]. MUC1 is considered to be one of the epithelial markers of porcine embryo pluripotent cells [20]. A decrease in MUC1 gene expression in the endometrium is responsible for a decrease in the rate of embryo implantation in humans [21].

The KITLG gene (KIT ligand), exhibiting increased expression in the OEC in vitro culture, is related to the development of, among others, stem cell or germ cell populationin reproductive organs, and its mutations may lead to porcine infertility [22].Its role is also described in maintaining the viability of primary germ cells. Studies of porcine uteri suggest that it also has a role in uterine intracellular communication [23].

The family of $\mathrm{ABC}$ transporters, to which belongs the $A B C A 1$ gene (ATP binding cassette subfamily A member 1) shows an involvement in the metabolism of steroids and fetal development, which is important in gametogenesis and folliculogenesis [24].

The bovine oviduct epithelial cells (BOECs) were examined for the effect of increased NEFA concentration. These studies showed that elevated NEFAs had an effect on the metabolism of BOECs. Additionally, the study suggests that the fallopian tube can significantly influence the conditions of early embryo development [25].

Significant changes in gene expression observed in our in vitro cultured OECs may indicate their potential role as markers of OECs development in pigs. Thanks to advanced molecular analyses we can get closer to a deeper understanding of the mechanisms controlling the changes in the epithelium of the fallopian tube and its correlation with the reproductive cells, both male and female.

\section{Acknowledgements}

Publication of this article was made possible by grant number UMO-2016/21/B/NZ9/03535 from Polish National Centre of Science. 


\section{Corresponding author}

Bartosz Kempisty PhD, Department of Histology and Embryology, Department of Anatomy, Poznań University of Medical Sciences, 6 Święcickiego St., 60-781 Poznań, Poland Tel./Fax: +48 618546418 /+4861 8546440, e-mail: bkempisty@ump.edu.pl.

\section{Conflict of interest statement}

The authors declare they have no conflict of interest.

\section{References}

1. Kranc W, Budna J, Chachuła A, Borys S, Bryja A, Rybska M, Ciesiółka S, Sumelka E, Jeseta M, Brüssow KP, Bukowska D, Antosik P, Bruska M, Nowicki M, Zabel M, Kempisty B. "Cell Migration" Is the Ontology Group Differentially Expressed in Porcine Oocytes Before and After In Vitro Maturation: A Microarray Approach. DNA Cell Biol. 2017;36:273-82 DOI:10.1089/dna.2016.3425.

2. Orisaka M, Tajima K, Tsang BK, Kotsuji F. Oocyte-granulosa-theca cell interactions during preantral follicular development. J Ovarian Res. 2009;2:9; DOI:10.1186/1757-2215-2-9.

3. Coy P, García-Vázquez FA, Visconti PE, Avilés M. Roles of the oviduct in mammalian fertilization. Reproduction. 2012;144:649-60; DOI:10.1530/REP-12-0279.

4. Kölle S, Dubielzig S, Reese S, Wehrend A, König P, Kummer W. Ciliary transport, gamete interaction, and effects of the early embryo in the oviduct: ex vivo analyses using a new digital videomicroscopic system in the cow. Biol Reprod. 2009;81:267-74; DOI:10.1095/ biolreprod.108.073874

5. Tokuhiro K, Ikawa M, Benham AM, Okabe M. Protein disulfide isomerase homolog PDILT is required for quality control of sperm membrane protein ADAM3 and male fertility [corrected]. Proc Natl Acad Sci U S A. 2012;109:3850-5; DOI:10.1073/pnas.1117963109.

6. Ohtsu A, Tanaka H, Seno K, Iwata H, Kuwayama T, Shirasuna K. Palmitic acid stimulates interleukin-8 via the TLR4/NF- $\kappa B / R O S$ pathway and induces mitochondrial dysfunction in bovine oviduct epithelial cells. Am Reprod Immunol. 2017;77:1-10; DOI:10.1111/aji.12642.

7. Huang DW, Sherman BT, Tan Q, Collins JR, Alvord WG, Roayaei J, Stephens R, Baseler MW, Lane HC, Lempicki RA. The DAVID Gene Functional Classification Tool: a novel biological module-centric algorithm to functionally analyze large gene lists. Genome Biol. 2007;8:R183 DOI:10.1186/gb-2007-8-9-r183.

8. Walter W, Sánchez-Cabo F, Ricote M. GOplot: An R package for visually combining expression data with functional analysis. Bioinformatics. 2015;31:2912-4; DOI:10.1093/bioinformatics/btv300.

9. von Mering C, Jensen LJ, Snel B, Hooper SD, Krupp M, Foglierini M, Jouffre N, Huynen MA, Bork P. STRING: known and predicted protein-protein associations, integrated and transferred across organisms. Nucleic Acids Res. 2004;33:D433-7; DOI:10.1093/nar/gki005.

10. LeBlanc SJ. Interactions of metabolism, inflammation, and reproductive tract health in the postpartum period in dairy cattle. Reprod Domest Anim. 2012;47 Suppl 5:18-30; DOI:10.1111/j.1439-0531.2012.02109.x

11. Jungheim ES, Macones GA, Odem RR, Patterson BW, Lanzendorf SE, Ratts VS, Moley KH. Associations between free fatty acids, cumulus oocyte complex morphology and ovarian function during in vitro fertilization Fertil Steril. 2011;95:1970-4; DOI:10.1016/j.fertnstert.2011.01.154.

12. Van Hoeck V, Sturmey RG, Bermejo-Alvarez P, Rizos D, Gutierrez-Adan A, Leese HJ, Bols PEJ, Leroy JLMR. Elevated Non-Esterified Fatty Acid Concentrations during Bovine Oocyte Maturation Compromise Early Embryo Physiology. PLoS One. 2011;6:e23183; DOI:10.1371/journal. pone.0023183.

13. Jordaens L, Arias-Alvarez M, Pintelon I, Thys S, Valckx S, Dezhkam Y, Bols PEJ, Leroy JLMR. Elevated non-esterified fatty acid concentrations hamper bovine oviductal epithelial cell physiology in three different in vitro culture systems. Theriogenology. 2015;84:899-910; DOI:10.1016/j. theriogenology.2015.05.030

14. Huang A, Isobe N, Obitsu T, Yoshimura Y. Expression of lipases and lipid receptors in sperm storage tubules and possible role of fatty acids in sperm survival in the hen oviduct. Theriogenology. 2016;85:1334-42 DOI:10.1016/j.theriogenology.2015.12.020.

15. Lee Y, Lee H, Park B, Elahi F, Lee J, Lee ST, Park CK, Hyun SH, Lee E. Alpha-linolenic acid treatment during oocyte maturation enhances embryonic development by influencing mitogen-activated protein kinase activity and intraoocyte glutathione content in pigs. J Anim Sci. 2016;94:325563; DOI:10.2527/jas.2016-0384.

16. Yu YH, Wu SC, Cheng WTK, Mersmann HJ, Ding ST. Ectopic expression of porcine peroxisome proliferator-activated receptor $\delta$ regulates adipogenesis in mouse myoblasts. J Anim Sci. 2008;86:64-72; DOI:10.2527/ jas.2007-0399.
17. Ruberte E, Friederich V, Morriss-Kay G, Chambon P. Differential distribution patterns of CRABP-I and CRABP-II transcripts during mouse embryogenesis. Development. 1992;115:973-89.

18. Hornemann T, Richard S, Rütti MF, Wei Y, von Eckardstein A. Cloning and Initial Characterization of a New Subunit for Mammalian Serine-palmitoyltransferase. J Biol Chem. 2006;281:37275-81; DOI:10.1074/jbc. M608066200

19. Gibier J-B, Hémon B, Fanchon M, Gaudelot K, Pottier N, Ringot B, Van Seuningen I, Glowacki F, Cauffiez C, Blum D, Copin M-C, Perrais M, Gne$\mathrm{mmi}$ V. Dual role of MUC1 mucin in kidney ischemia-reperfusion injury: Nephroprotector in early phase, but pro-fibrotic in late phase. Biochim Biophys Acta - Mol Basis Dis. 2017;1863:1336-49; DOI:10.1016/j. bbadis.2017.03.023.

20. Hall VJ, Jacobsen J V, Rasmussen MA, Hyttel P. Ultrastructural and molecular distinctions between the porcine inner cell mass and epiblast reveal unique pluripotent cell states. Dev Dyn. 2010;239:2911-20; DOI:10.1002/dvdy.22424.

21. Wu F, Chen X, Liu Y, Liang B, Xu H, Li TC, Wang CC. Decreased MUC1 in endometrium is an independent receptivity marker in recurrent implantation failure during implantation window. Reprod Biol Endocrinol. 2018;16:60; DOI:10.1186/s12958-018-0379-1.

22. Hadjiconstantouras C, Sargent CA, Skinner TM, Archibald AL, Haley CS, Plastow GS. Characterization of the porcine KIT ligand gene: expression analysis, genomic structure, polymorphism detection and association with coat colour traits. Anim Genet. 2008;39:217-24 DOI:10.1111/j.1365-2052.2008.01708.x

23. Zhang Z, Anthony R V. Porcine stem cell factor/c-kit ligand: its molecular cloning and localization within the uterus. Biol Reprod. 1994;50:95-102.

24. Wang H, Zhou L, Gupta A, Vethanayagam RR, Zhang Y, Unadkat JD, Mao Q. Regulation of BCRP/ABCG2 expression by progesterone and 17beta-estradiol in human placental BeWo cells. Am J Physiol Endocrinol Metab. 2006;290:E798-807; DOI:10.1152/ajpendo.00397.2005.

25. Jordaens L, Van Hoeck V, Maillo V, Gutierrez-Adan A, Marei WFA, Vlaeminck B, Thys S, Sturmey RG, Bols PEJ, Leroy JLMR. Maternal metabolic stress may affect oviduct gatekeeper function. Reproduction. 2017;153:759-73; DOI:10.1530/REP-16-0569. 